\title{
High Momentum Hadron and Jet Production in Photon-Photon Collisions at LEP2
}

\author{
Attila Krasznahorkay Jr. ${ }^{1,2}$ for the OPAL collaboration \\ 1- University of Debrecen - Dept. of Experimental Physics \\ 4010, Debrecen, Egyetem tér 1. - Hungary \\ 2- CERN - PH Dept. \\ CH-1211, Geneva 23 - Switzerland
}

\begin{abstract}
The inclusive production of charged hadrons $\left(\mathrm{e}^{+} \mathrm{e}^{-} \rightarrow \mathrm{e}^{+} \mathrm{e}^{-}+X\right)$ and jets $\left(\mathrm{e}^{+} \mathrm{e}^{-} \rightarrow\right.$ $\mathrm{e}^{+} \mathrm{e}^{-}+$jet $+\mathrm{X}$ ) have been studied in collisions of quasi-real photons radiated by the LEP beams at $\mathrm{e}^{+} \mathrm{e}^{-}$centre-of-mass energies $\sqrt{s_{\text {ee }}}$ from 183 to $209 \mathrm{GeV}$. The differential cross-sections measured as a function of the transverse momentum and pseudorapidity of the hadrons and jets are compared to theoretical calculations in next-to-leading order of the strong coupling constant.
\end{abstract}

\section{Introduction}

We have studied the inclusive production of jets in collisions of two quasi-real photons at $\mathrm{e}^{+} \mathrm{e}^{-}$centre-of-mass energies, $\sqrt{s_{\mathrm{ee}}}$, from 189 to $209 \mathrm{GeV}$, with a total integrated luminosity of $593 \mathrm{pb}^{-1}$ [2], and the inclusive production of charged hadrons at $\sqrt{s_{\mathrm{ee}}}$ from 183 to $209 \mathrm{GeV}$ with a total integrated luminosity of $612.8 \mathrm{pb}^{-1}[3]$. The data has been collected with the OPAL $[4,5,6]$ detector.

Hadronic interactions of two photons lead to the production of hadrons whose properties in Quantum Chromo Dynamics (QCD) depend on the underlying partonic processes, as well as on the way in which the partons are transformed into observable hadrons (fragmentation). In the measurement of hadron production this transformation is described by fragmentation functions obtained from fits to data. Jet observables on the other hand are constructed with the aim of minimising the difference between the observed quantity on parton and on hadron level. The remaining discrepancies (hadronisation corrections) are studied using hadronisation models as implemented in MC event generators. The studies of hadron and jet production therefore complement each other in the attempt to unveil the underlying partonic processes in the description of hadronic photon-photon interactions.

\section{Analysis}

For the hadron cross section measurements the tracks of the charged hadrons are reconstructed in the central tracking chamber. To select events coming from photon-photon interactions, standard cuts on reconstructed quantities are applied. Selected events have to have at least six tracks, passing tight quality cuts. Single- and double-tagged events are rejected by applying a $60 \mathrm{GeV}$ cut on the total measured energy in the forward detectors. Beam-gas and beam-wall backgrounds are reduced by requiring the primary vertex to have a radial distance less than $2 \mathrm{~cm}$ from the beam axis and a $z$-distance of less than $3 \mathrm{~cm}$ from the interaction point.

The inclusive jet cross section analysis uses the $k_{\perp}$ algorithm $[7,8]$ to reconstruct jets from photon-photon interactions. Since high $p_{\mathrm{T}}$ inclusive jet events from photon-photon 
interactions are difficult to separate from hadronic $\mathrm{Z}$ decays, especially at high momenta, a likelihood event selection [9] was used to separate signal and background events. The performance of the event selection is demonstrated in Figure 1.

The measured transverse momentum and pseudorapidity distributions have to be corrected for the losses due to event, track and jet selection cuts, the acceptance and the resolution of the detector. This is done using the Monte Carlo signal events which were processed by the full detector simulation and reconstruction chain. The correction factors are calculated as the binby-bin ratio of the generated and reconstructed Monte Carlo distributions. This method only yields reliable results if migration effects between bins due to the finite resolution of the measurements are small. The bins in the differential distributions have therefore been chosen to be significantly larger than the detector resolution, obtained from the Monte Carlo simulation.

NLO calculations do not take into account the possibility of an underlying event,

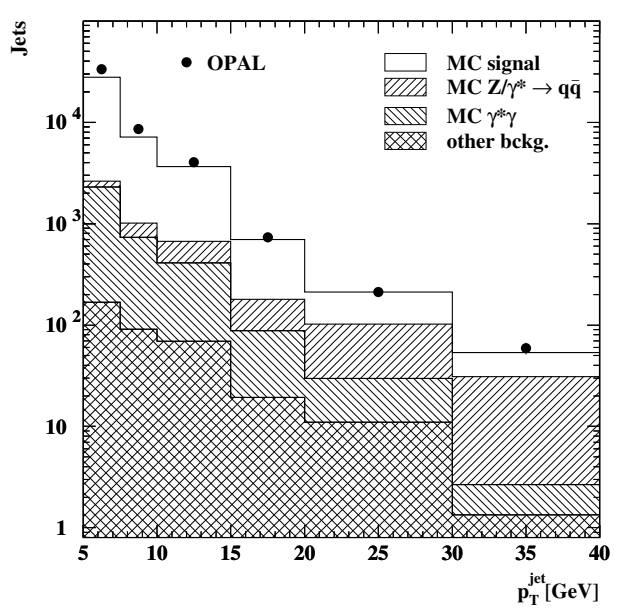

Figure 1: Number of jets in each $p_{\mathrm{T}}^{\text {jet }}$ bin after event selection which leads to an increased energy flow and therefore a larger cross-section above any given threshold in transverse momentum. In the inclusive jet analysis PYTHIA [10], which uses multiple parton interactions (MIA) to model this effect, was used to study either considering or leaving out multiple parton interactions for the signal MC. At the lowest transverse momenta considered, the signal MC cross-section increases by up to $20 \%$ when including MIA. This effect reduces to less than $10 \%$ for transverse momenta larger than $7 \mathrm{GeV}$.

The measured cross-sections are compared to NLO QCD calculations which describe cross-sections for partons, while the experimental cross-sections are presented for hadrons. Hadronisation corrections have also been estimated with PYTHIA. For inclusive jets the correction at $p_{\mathrm{T}}^{\text {jet }}=5 \mathrm{GeV}$ is about $-15 \%$. The correction decreases with increasing $p_{\mathrm{T}}^{\text {jet }}$ and is below $-5 \%$ for $p_{\mathrm{T}}^{\text {jet }}>20 \mathrm{GeV}$.

\section{Differential cross-sections}

To facilitate a comparison with a recent measurement by the L3 collaboration [11], the inclusive jet cross section measurement was performed with kinematic conditions such as the ones used in the L3 analysis. Figure 2 shows the resulting inclusive jet differential cross section. It shows the total statistical and systematical uncertainties added in quadrature where larger than the marker size. The uncertainty band on the NLO calculation shows the uncertainty associated to the variation of the renormalisation scale.

Both PYTHIA 6.221 and the NLO calculation achieve a good description of the data, with the exception of the lowest bin in $p_{\mathrm{T}}^{\mathrm{jet}}$, where the NLO calculation is too low. While the L3 data points are compatible with the present measurement, they lie below the OPAL 


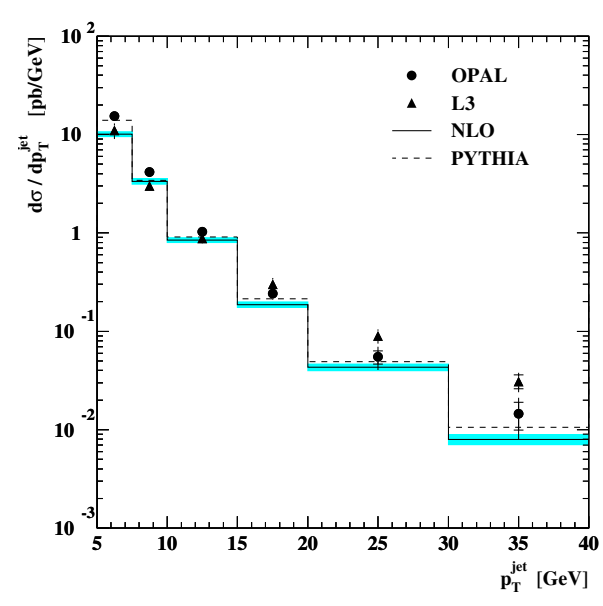

Figure 2: Inclusive jet differential cross section, $d \sigma / d p_{\mathrm{T}}^{\text {jet }}$, for all jets with $\left|\eta^{\text {jet }}\right|<1.0$ compared to the results of the L3 collaboration, NLO and PYTHIA predictions.
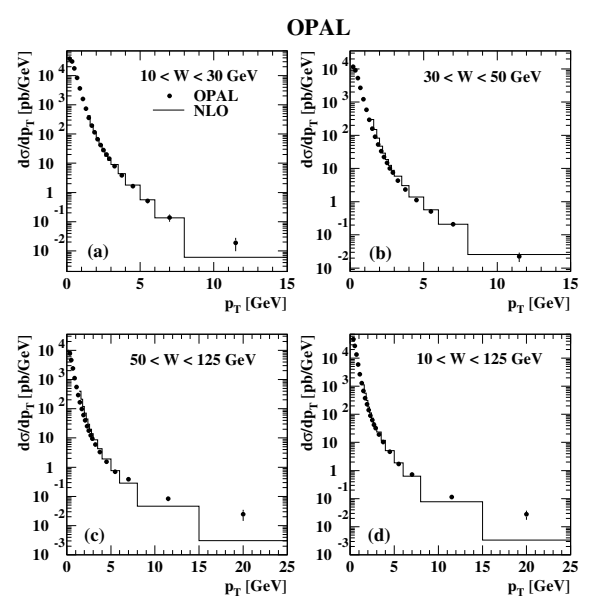

Figure 3: Differential inclusive charged hadron production cross-sections $d \sigma / d p_{\mathrm{T}}$ for $|\eta|<1.5$ in the indicated $W$ ranges.

data points at low $p_{\mathrm{T}}^{\text {jet }}$ and above the OPAL data points at high $p_{\mathrm{T}}^{\text {jet }}$, and there is a discrepancy in shape between the L3 data and the NLO calculation. This difference in shape has been reported in the L3 publication and leads to a significant disagreement between the L3 measurement and the NLO calculation at the highest $p_{\mathrm{T}}^{\text {jet }}$ of up to $50 \mathrm{GeV}$ studied in [11]. The OPAL analysis [2] finds the region of $p_{\mathrm{T}}^{\text {jet }}>40 \mathrm{GeV}$ to be dominated by background and hence no measurement is presented for this region.

The differential inclusive cross sections $d \sigma / d p_{\mathrm{T}}$ for charged hadrons in four ranges of the hadronic invariant mass $W$ are shown in Figure 3. For the cross section calculation a minimum $p_{\mathrm{T}}$ of $1.5 \mathrm{GeV}$ is required to ensure the validity of the perturbative QCD calculation. The NLO calculation achieves a good general description of the data, except for transverse momenta greater than about $10 \mathrm{GeV}$, which can be reached in the highest $W$ range only.

Figure 4 (a) and (b) show the differential cross-section $d \sigma / d p_{\mathrm{T}}$ for charged hadrons

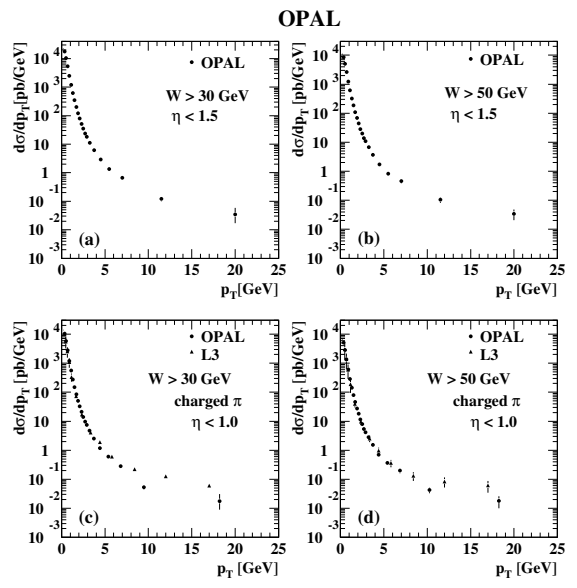

Figure 4: Differential inclusive charged hadron production cross-sections $d \sigma / d p_{\mathrm{T}}$ in the indicated $\eta$ and $W$ ranges. for $W>30 \mathrm{GeV}$ and $W>50 \mathrm{GeV}$. To facilitate a comparison with a recent measurement by L3 [12] of charged pions, the measure- 
ment has been done in the pseudorapidity range $|\eta|<1.0$ in (c) and (d). The OPAL data shown in Figure 4 (c) and (d) have been scaled to account for the reduced $|\eta|$ range and for the fraction of charged pions of all charged hadrons using MC simulations. From this comparison it is evident that the distributions measured by OPAL fall more rapidly towards high transverse momenta than those measured by L3, leading to a disagreement between the two experiments at high transverse momenta and a better description of the OPAL data by NLO QCD than is the case for the L3 data.

\section{References}

[1] Slides: http://indico. cern. ch/contributionDisplay . py? contribId=213\&sessionId=6\&conf $I d=9499$

[2] G. Abbiendi et al. [OPAL Collaboration], "Inclusive Jet Production in Photon-Photon Collissions at $\sqrt{s_{e e}}$ from 189 to $209 \mathrm{GeV}$ ", Submitted to Phys. Lett. B

[3] G. Abbiendi et al. [OPAL Collaboration], "Inclusive Production of Charged Hadrons in Photon-Photon Collisions", Accepted by Phys. Lett. B [arXiv:hep-ex/0612045v1]

[4] K. Ahmet et al. [OPAL Collaboration], Nucl. Instrum. Meth. A 305 (1991) 275.

[5] G. Abbiendi et al. [OPAL Collaboration], Eur. Phys. J. C 14 (2000) 373 [arXiv:hep-ex/9910066].

[6] G. Abbiendi et al. [OPAL Collaboration], Eur. Phys. J. C 33 (2004) 173 [arXiv:hep-ex/0309053].

[7] S. Catani, Y. L. Dokshitzer, M. H. Seymour and B. R. Webber, Nucl. Phys. B 406 (1993) 187.

[8] S. D. Ellis and D. E. Soper, Phys. Rev. D 48 (1993) 3160 [arXiv:hep-ph/9305266].

[9] D. A. Karlen, Comput. Phys. 12:4 (1998) 380.

[10] T. Sjostrand, Comput. Phys. Commun. 82 (1994) 74

[11] P. Achard et al. [L3 Collaboration], Phys. Lett. B 602 (2004) 157 [arXiv:hep-ex/0410012].

[12] P. Achard et al. [L3 Collaboration], Phys. Lett. B 554 (2003) 105 [arXiv:hep-ex/0301025]. 